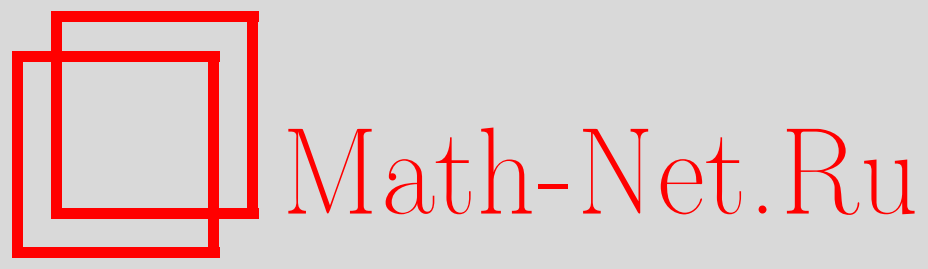

В. А. Васильев, О топологических инвариантах вещественных алгебраических функции, Функи. анализ и его прил., 2011, том 45, выпуск 3, 4-15

DOI: https://doi.org/10.4213/faa3048

Использование Общероссийского математического портала Math$\mathrm{Net.Ru} \mathrm{подразумевает,} \mathrm{что} \mathrm{вы} \mathrm{прочитали} \mathrm{и} \mathrm{согласны} \mathrm{с} \mathrm{пользователь-}$ ским соглашением

http://www . mathnet.ru/rus/agreement

Параметры загрузки:

IP: 18.234 .197 .8

26 апреля 2023 г., 13:30:39

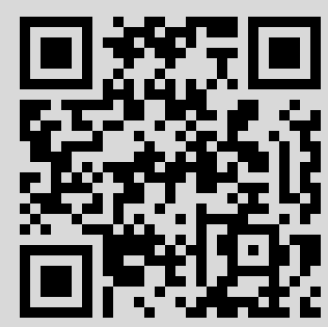


Функционалъный анализ и его приложения

2011, т. 45, вып. 3, с. 4-15

УДК 515.16

\title{
О топологических инвариантах вещественных алгебраических функций*
}

\author{
(c) 2011. В. А. ВАСИЛЬЕВ
}

\section{Памяти Владимира Игоревича Арнольда}

\begin{abstract}
Мы рассматриваем естественное накрытие, отвечающее за сложность ветвления корней общего вещественного полиномиального уравнения, и вычисляем гомологии его базы, а для уравнений степени $\leqslant 5$ полностью описываем топологию этой базы.
\end{abstract}

Ветвление значений общей комплексной $d$-значной целой алгебраической функции $x=F\left(a_{1}, \ldots, a_{d}\right)$, заданной условием

$$
x^{d}+a_{1} x^{d-1}+\cdots+a_{d-1} x+a_{d}=0,
$$

возникает на дискриминантном множестве $\Sigma_{\mathbb{C}} \subset \mathbb{C}^{d}$, состоящем из тех наборов коэффициентов $\left(a_{1}, \ldots, a_{d}\right)$, при которых полином $(1)$ имеет кратные корни, см. [1], [5]. Дополнение до этого множества в $\mathbb{C}^{d}$ является базой двух стандартных накрытий: $d$-листного и $d$ !-листного. Фундаментальная группа этого дополнения действует на наборах корней уравнения (1) и порождает всю группу перестановок корней. В. И. Арнольд использовал гомологии этого дополнения $\mathbb{C}^{d} \backslash \Sigma_{\mathbb{C}}$ как препятствие к индуцированию алгебраических функций друг из друга. Также изучение этих гомологий доставляет нижние оценки на род Швариа соответствующих накрытий, т. е. на минимальное число открытых подмножеств, покрывающих эту базу, над каждым из которых накрытие имеет непрерывное сечение, см. [7], [8]. В [8], [9] эти оценки использованы для изучения топологической сложности приближенного решения общего уравнения (1).

Если ограничиться только вещественными уравнениями вида (1), то аналогичную роль будут играть накрытия, определенные на дополнении к некоторому подмножеству вещественной коразмерности 2 в пространстве $\mathbb{R}^{d}$ таких уравнений. А именно, это подмножество $\Upsilon$ состоит из полиномов, имеющих либо вещественный корень кратности $\geqslant 3$, либо пару мнимых комплексносопряженных корней кратности $\geqslant 2$. При $d=4$ это множество в пространстве приведенных (т. е. с $a_{1}=0$ ) полиномов (1) задается тремя ветвями кривых, уходящими из начала координат на бесконечность и выделенными в левой части рис. 1: двумя ветвями ребра возврата ласточкина хвоста (см., например, [2]) и продолжением его линии самопересечения.

Монодромия этих накрытий порождает не всю группу перестановок $d$ корней, а лишь подгруппу четных перестановок. Как и в комплексном случае, топология этих накрытий доставляет нижние оценки на число ветвлений алгоритмов,

*Работа поддержана грантом НШ-8462.2010.1 Президента РФ для поддержки ведущих научных школ. 

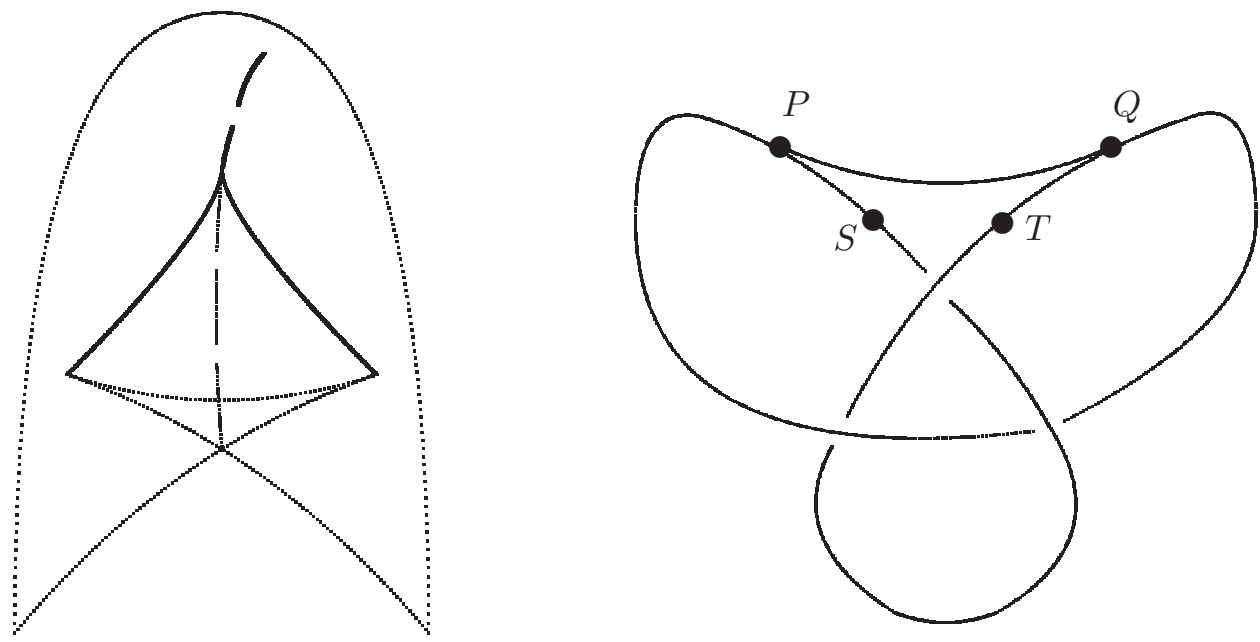

Рис. 1. Множество существенного ветвления для $d=4$ и 5

решающих вещественные уравнения (1); уже при $d=3$ это вычисление доказывает неизбежность таких ветвлений.

В $\S 4$ мы вычисляем целочисленные гомологии всех пространств $\mathbb{R}^{d} \backslash \Upsilon$.

$\mathrm{B} \S 2$ эти накрытия описаны в простейших случаях $d=3$ и 4 . В 33 мы находим топологический тип множества $\Upsilon$ (как вложенного подмножества в $\mathbb{R}^{d}$ ) для $d=5$. А именно, мы сводим эту задачу к изучению пересечения этого множества со сферой $S^{3}$, охватывающей начало координат в пространстве приведенных полиномов

$$
x^{5}+A x^{3}+B x^{2}+C x+D
$$

и доказываем следующую теорему.

Теорема 1. При $d=5$ пересечение множества $\Upsilon$ c $S^{3}$ изотопно фигуре, изображенной в правой части рис. 1. При этом

- точки $P$ и $Q$ соответствуют полиномам с четырехкратными корнями;

- кратчайший криволинейный интервал $(P, Q)$ состоит из полиномов $c$ тройным корнем, лежащим между двумя другими вещественными корнями;

- более длинный криволинейный интервал $(P, Q)$, не содерэсащий точек $S$ и $T$, состоит из полиномов с двукратными мнимыми корнями;

- точки $S$ и $Т$ обозначают два полинома с двукратным и трехкратным вещественными корнями у каждого;

- интерваль $(P, S)$ и $(Q, T)$ состоят из полиномов с одним трехкратным корнем, бо́льшим (соответственно меньшим) двух других вещественньх корней;

- криволинейный интервал $(S, T)$, не содержащий точек $P$ u $Q$, состоит из полиномов с трехкратным вещественным корнем и парой мнимых.

Характер поведения трех ветвей множества $\Upsilon$ вблизи точек $P$ и $Q$ повторяет поведение вблизи начала трех выделенных ветвей в левой части рис. 1. 
Следствие 1. Дополнение до множества $\Upsilon$ в $S^{3}$ гомотопически эквивалентно одномерному комплексу.

Замечание 1. Правая часть рис. 1 является расширением рис. 28 из разд. 2.5 в [3] (на котором подробно изображена верхняя центральная часть этой части рис. 1). Принципиальная деталь этого расширения - поведение «фантомной» линии ветвления, соответствующей полиномам с мнимыми совпадающими корнями, по отношению к действительной части.

\section{$\S 1$. Основное накрытие $\Theta_{d}$}

Над пространством $\mathbb{C}^{d} \backslash \Sigma_{\mathbb{C}}$ комплексных полиномов (1), все корни которых различны, имеется очевидное $d$-листное накрытие

$$
\varphi_{d}: M_{\mathbb{C}}^{d} \rightarrow \mathbb{C}^{d} \backslash \Sigma_{\mathbb{C}},
$$

слой которого над полиномом $f$ - это множество пар, состоящих из полинома $f$ и одного из его корней.

Определение 1. Множеством существенного ветвления $\Upsilon$ в пространстве $\mathbb{R}^{d}$ вещественных полиномов (1) называется объединение множества полиномов с хотя бы одним вещественным корнем кратности $\geqslant 3$ и множества полиномов с хотя бы одной парой сопряженных мнимых корней кратности $\geqslant 2$. Последние два множества обозначаются соответственно через \&

Очевидно, все эти множества имеют коразмерность 2 в $\mathbb{R}^{d}$.

Предложение 1. Ограничение накрытия $\varphi_{d}$ на множество $\mathbb{R}^{d} \backslash \Sigma_{\mathbb{C}}$ продолжается до накрытия над множеством $\mathbb{R}^{d} \backslash \Upsilon$.

Действительно, добавочное множество $\left(\mathbb{R}^{d} \cap \Sigma_{\mathbb{C}}\right) \backslash \Upsilon$, на которое требуется продолжить накрытие $\varphi_{d}$, состоит из полиномов, имеющих некоторое количество вещественных корней кратности ровно 2 . Если такой полином $f_{0}$ имеет ровно $k$ двойных корней $q_{1}<\cdots<q_{k}$, то множество $\mathbb{R}^{d} \cap \Sigma_{\mathbb{C}}$ вблизи точки $f_{0}$ объемлемо диффеоморфно прямому произведению пространства $\mathbb{R}^{d-k}$ и объединения координатных плоскостей в $\mathbb{R}^{k}$. При движении в окрестности такого полинома $f_{0} \in \mathbb{R}^{d}$ пары близких вещественных корней могут сливаться и уходить в комплексную область. Над такой окрестностью $U$ определим $2 k$-листное тривиальное накрытие, т. е. произведение $U$ на множество из $2 k$ точек $r_{1}^{+}, r_{1}^{-}, \ldots, r_{k}^{+}, r_{k}^{-}$. Пусть $f \in U$ не принадлежит $\Sigma_{\mathbb{C}}$ и для некоторого $i \in\{1, \ldots, k\}$ полином $f$ имеет два вещественных корня, получившихся распадением корня $q_{i}$ полинома $f_{0}$. Тогда отождествим точку $(f$, бо́льший из этих корней $) \in M_{\mathbb{C}}^{d}$ с точкой $f \times r_{i}^{+}$этого произведения, а точку $(f$, меньший из этих корней $)-$ с $f \times r_{i}^{-}$. Аналогично, если $f$ имеет два мнимых корня, получившихся распадением $q_{i}$, то отождествим точку $\left(f\right.$, корень с положительной мнимой частью) $\in M_{\mathbb{C}}^{d}$ с $f \times r_{i}^{+}$, а точку $(f$, корень с отрицательной $)-\mathrm{c} f \times r_{i}^{-}$. Эта склейка продолжает $2 k$ листов нашего накрытия $\varphi_{d}$ на всю окрестность $U$, а оставшиеся $d-2 k$ листов продолжаются туда же очевидным образом. Такие продолжения согласованы для всех точек множества $\left(\mathbb{R}^{d} \cap \Sigma_{\mathbb{C}}\right) \backslash \Upsilon$.

Обозначим это продолженное накрытие так: $\Theta_{d}: M_{\mathbb{R}}^{d} \rightarrow \mathbb{R}^{d} \backslash \Upsilon$.

Замечание 2. Вот другое описание этого же накрытия. Каждая локальная (вблизи $f_{0}$ ) неприводимая компонента множества $\left(\mathbb{R}^{d} \cap \Sigma_{\mathbb{C}}\right) \backslash \Upsilon$ неособа и 
трансверсально ориентирована в ту сторону, в которой соответствующий двукратный корень распадается в два вещественных. Определим гладкое векторное поле $v$ в $\mathbb{R}^{d}$, нулевое вне малой окрестности этого множества и в том числе на $\Upsilon$, трансверсальное ко всем этим локальным компонентам и пересекающее их в положительном направлении. Затем сдвинем $\mathbb{R}^{d} \backslash \Upsilon$ в комплексной области, переводя каждую точку $f$ в $f+i v(f)$. При соблюдении должных ограничений на длину $v$ это отображение вложит все $\mathbb{R}^{d} \backslash \Upsilon$ в $\mathbb{C}^{d} \backslash \Sigma_{\mathbb{C}}$. Накрытие $\Theta_{d}$ эквивалентно индуцированному этим сдвигом из накрытия (3).

Накрытие $\Theta_{d}$ определяет представление монодромии $m_{d}: \pi_{1}\left(\mathbb{R}^{d} \backslash \Upsilon\right) \rightarrow S(d)$, где $S(d)$ - группа перестановок корней отмеченного полинома (в качестве которого удобно взять какой-нибудь полином с $d$ разными вещественными корнями). При этом описание накрытия $\Theta_{d}$, данное в замечании 2 , в некотором смысле удобнее исходного, так как оно фиксирует не только представление монодромии в группу перестановок $d$ корней, но и его поднятие $\bar{m}_{d}: \pi_{1}\left(\mathbb{R}^{d} \backslash \Upsilon\right) \rightarrow B r(d)$ в гpynny кос из d нитей (определение которой см., например, в [2], [10]).

Предложение 2. Для любого $d$ образ представления $m_{d}: \pi_{1}\left(\mathbb{R}^{d} \backslash \Upsilon\right) \rightarrow S(d)$ совпадает с подгруппой $A(d)$ четных перестановок. Образ поднятия $\bar{m}_{d}$ этого представления лежит в подгруппе кос нулевой закрученности.

Доказательство. Назовем полином $f \in \mathbb{R}^{d} \backslash \Sigma_{\mathbb{C}}$ типичным, если никакое вещественное число не является вещественной частью более чем двух его корней. Для любого такого $f$ пронумеруем все его корни в порядке возрастания вещественных частей, а в случае их совпадения - в порядке возрастания мнимых. Имеется ровно два вида перестроек типичных полиномов, возникающих в путях общего положения в пространстве $\mathbb{R}^{d} \backslash \Upsilon$ и приводящих к скачкообразному изменению этой нумерации листов накрытия $M_{\mathbb{R}}^{d}$ над $f$ : совпадение вещественных частей одного вещественного и двух мнимых корней и совпадение вещественных частей двух пар мнимых корней. (Отметим, что слияние двух вещественных корней и их выход в комплексную область или обратное действие не являются перестройками, приводящими к такой перенумерации.) Каждая из этих перестроек определяет четную перенумерацию корней и, более того, косу нулевой закрученности, являющуюся произведением двух элементарных кос противоположного знака. С другой стороны, действием группы монодромии нашего накрытия легко реализуются любые циклические перестановки троек соседних элементов $(i-1, i, i+1) \mapsto(i+1, i-1, i)$ и перестановки пар соседних элементов $((i, i+1), \ldots,(j, j+1)) \mapsto((j, j+1), \ldots,(i, i+1))$. Действительно, для перестановки первого типа надо внутри множества полиномов с $d$ вещественными корнями подойти достаточно близко к полиному, у которого корни с номерами $i-1, i, i+1$ сливаются вместе, и обойти в $\mathbb{R}^{d}$ вокруг содержащего его страта полиномов с трехкратными корнями. Чтобы реализовать перестановку второго типа, надо столкнуть соответствующие пары корней, вывести их в комплексную область и простейшим способом поменять там местами. Перестановок этих двух типов достаточно, чтобы породить всю группу $A(d)$.

В [8] фактически показано (хотя явно сформулировано лишь позднее в [9]), что топологическая сложность (т. е. минимальное число ветвлений алгоритмов) приближенного вычисления одного корня любого комплексного полинома (1) оценивается снизу уменьшенным на 1 родом Шварца накрытия (3). Аналогичное утверждение (практически вместе с доказательством) переносится на 
задачу о приближенном решении любого вещественного уравнения вида (1) (разумеется, эта задача актуальна только для нечетных $d$ ).

Предложение 3 (ср. [8]). Для любого нечетного $d$ u компакта $D \subset \mathbb{R}^{d}$, содержсащего окрестность начала координат, найдется $\varepsilon(D)>0$, такое, что число ветвлений (операторов IF) любого алгебраического алгоритма, вычисляющего с погрешностью $\leqslant \varepsilon$ какой-нибудь вещественный корень всякого уравнения (1) с $\left(a_{1}, \ldots, a_{d}\right) \in D$, не менъше уменьшенного на 1 рода накрытия $\Theta_{d}$.

\section{§2. Первая редукция и простейшие примеры: $d=3$ и 4}

Группа $\mathbb{R}^{1}$ сдвигов аргумента свободно действует на пространстве полиномов (1) по правилу $t: f(x) \mapsto f(x-t)$, сохраняя всю стратификацию этого пространства по кратностям и вещественности корней. Каждая орбита этого действия единожды трансверсально пересекает подпространство, состоящее из полиномов (1) с $a_{1}=0$. Расслоение пространства всех полиномов на эти орбиты задает сохраняющий эту стратификацию диффеоморфизм пространства $\mathbb{R}^{d}$ на прямое произведение прямой $\mathbb{R}^{1}$ и этого подпространства $\mathbb{R}^{d-1}$. Поэтому достаточно рассматривать только это подпространство и ограничение накрытия $\Theta_{d}$ на $\mathbb{R}^{d-1} \backslash \Upsilon$.

2.1. Случай $\boldsymbol{d}=\mathbf{3}$. В этом случае мы получаем пространство полиномов $x^{3}+p x+q$. Оно разбито дискриминантной кривой $\left\{(p / 3)^{3}+(q / 2)^{2}=0\right\}$ на две неравные части, в меньшей из которых все полиномы имеют по три вещественных корня, а в большей - один. Множество $\Upsilon$ в этом пространстве представлено единственной точкой - началом координат. Легко проверить, что однократный обход вокруг этой точки, начинающийся и кончающийся в полиноме с тремя вещественными корнями, определяет циклическую перестановку этих корней. В частности, род Шварца накрытия $\Theta_{3}$ над $\mathbb{R}^{2} \backslash \Upsilon$ равен 2.

2.2. Случай $\boldsymbol{d}=\mathbf{4}$. В этом случае дискриминантное подмножество в $\mathbb{R}^{3}$ - это ласточкин хвост, см. левую часть рис. 1. Множество $\Upsilon \cap \mathbb{R}^{3}$ при этом состоит из трех незаузленных кривых, выходящих из начала координат и уходящих на бесконечность: двух ветвей ребра возврата $A_{2}$ и добавляемой части аналитического продолжения линии самопересечения хвоста; при этом видимая линия самопересечения, состоящая из полиномов, имеющих пару вещественных корней кратности 2 , не принадлежит множеству $\Upsilon$. Простейшие петли в $\mathbb{R}^{3} \backslash \Upsilon$, начинающиеся и кончающиеся в каком-нибудь полиноме с четырьмя вещественными корнями и охватывающие эти три кривые, задают следующие перестановки этих корней: $(1,2,3,4) \mapsto(2,3,1,4),(1,3,4,2)$ и $(3,4,1,2)$ соответственно. Очевидно, эти перестановки четны, зависимы и порождают группу $A(4)$. Род Шварца накрытия $\Theta_{4}$ над $\mathbb{R}^{3} \backslash \Upsilon$ не меньше 2 (что означает просто отсутствие у него глобальных сечений). Но он и не может превосходить 2, поскольку база этого накрытия гомотопически эквивалентна одномерному комплексу, см. [7].

Аналогично, из следствия 1 вытекает, что род накрытия $\Theta_{5}$ также равен 2. Это согласуется с результатом из [11] о том, что топологическая сложность приближенного решения общего уравнения (2) равна 1. С другой стороны, в [12] показано, что для уравнений седьмой степени эта сложность уже не меньше 2, поэтому случай $d=7$ является кандидатом на следующее повышение рода Шварца накрытия $\Theta_{d}$ (до тройки). 


\section{§3. Доказательство теоремы 1}

\section{1. Внутренняя топология множества существенного ветвления.} Группа $\mathbb{R}_{+}^{1}$ положительных растяжений аргумента действует на пространстве полиномов (1) по правилу

$$
\mathbb{R}_{+}^{1} \ni \lambda: f(x) \mapsto \lambda^{-d} f(\lambda x) .
$$

Это действие свободно на $\mathbb{R}^{d-1} \backslash 0$ и вновь сохраняет всю комбинаторную стратификацию множества полиномов. Каждая орбита этого действия единожды трансверсально пересекает границу $S^{d-2}$ любого шара с центром в $0 \in \mathbb{R}^{d-1}$, и мы получаем диффеоморфизм $\mathbb{R}^{d-1} \backslash 0 \simeq S^{d-2} \times \mathbb{R}_{+}^{1}$. Поэтому при $d=5$ достаточно рассматривать такую сферу $S^{3}$ в пространстве полиномов (2) и ее пересечение с множеством $\Upsilon$. В качестве такой сферы можно взять множество всех полиномов, все корни которых по модулю не превосходят единицы и по меньшей мере один из этих модулей равен единице; сумма всех этих корней должна равняться 0. (Эта сфера не является гладким подмножеством в $\mathbb{R}^{4}$, но мы рассматриваем ее как реализацию факторпространства $\left(\mathbb{R}^{d-1} \backslash 0\right) / \mathbb{R}_{+}^{1}$ с соответствующей гладкой структурой.)

Предложение 4. Пересечение множества существенного ветвления $\Upsilon \in$ $\mathbb{R}^{5}$ для общей алгебраической функиии 5-й степени со сферой $S^{3}$ в пространстве полиномов (2) гомеоморфно фигуре $\bigcirc$. При этом особые точки этого множества соответствуют полиномам с четырехкратными корнями.

Доказательство этого утверждения состоит в перечислении всевозможных неупорядоченных пятерок точек в единичном круге в $\mathbb{C}^{1}$, симметричных относительно комплексного сопряжения и имеющих нулевой центр тяжести, причем хотя бы одна из точек должна лежать на границе круга и либо какие-то три точки должны сливаться, либо имеются две сопряженные пары слившихся точек. Это перечисление приведено на рис. 2. При этом буквы $P, Q, S, T$ обозначают те же полиномы, что в теореме 1 и на рис. 1 ; смысл букв $L, K, X, V, W, Y$ будет объяснен позднее.

Остается понять, как этот граф $\bigcirc$ вложен в $S^{3}$.

3.2. Центральный симплекс. Самая интересная часть сферы $S^{3}-$ множество полиномов, имеющих 5 вещественных корней. Это множество канонически гомеоморфно стандартному трехмерному симплексу: если обозначить значения корней полинома через $r_{1}<r_{2}<\cdots<r_{5}$, то в качестве барицентрических координат точки симплекса, соответствующей этому полиному, можно взять числа $\alpha \equiv \frac{r_{2}-r_{1}}{r_{5}-r_{1}}, \beta \equiv \frac{r_{3}-r_{2}}{r_{5}-r_{1}}, \gamma \equiv \frac{r_{4}-r_{3}}{r_{5}-r_{1}}, \delta \equiv \frac{r_{5}-r_{4}}{r_{5}-r_{1}}$. Этот гомеоморфизм не является объемлемым диффеоморфизмом, см. рис. 3: например, вблизи вершин $\alpha=1$ и $\delta=1$ (или, в терминах рис. 2, точек $P$ и $Q$ ) этот симплекс диффеоморфен внутренней пирамидке ласточкина хвоста, а вблизи ребер $\{\gamma=\delta=0\}$, $\{\alpha=\beta=0\}$ и $\{\beta=\gamma=0\}-$ меньшей из двух областей, разделяемых поверхностью с полукубическим ребром возврата. Эти три ребра представлены на рис. 2 соответственно отрезками $[P S],[Q T]$ и $[P Q]$.

Остается понять, как заузлены оставшиеся два отрезка кривой $\Upsilon$.

Будем представлять себе нашу сферу $S^{3}$ как факторпространство пространства $\mathbb{R}^{4} \backslash 0$ полиномов вида (2) по действию (4). Заметим, что множество $\Upsilon \cap \mathbb{R}^{4} \backslash 0$ 


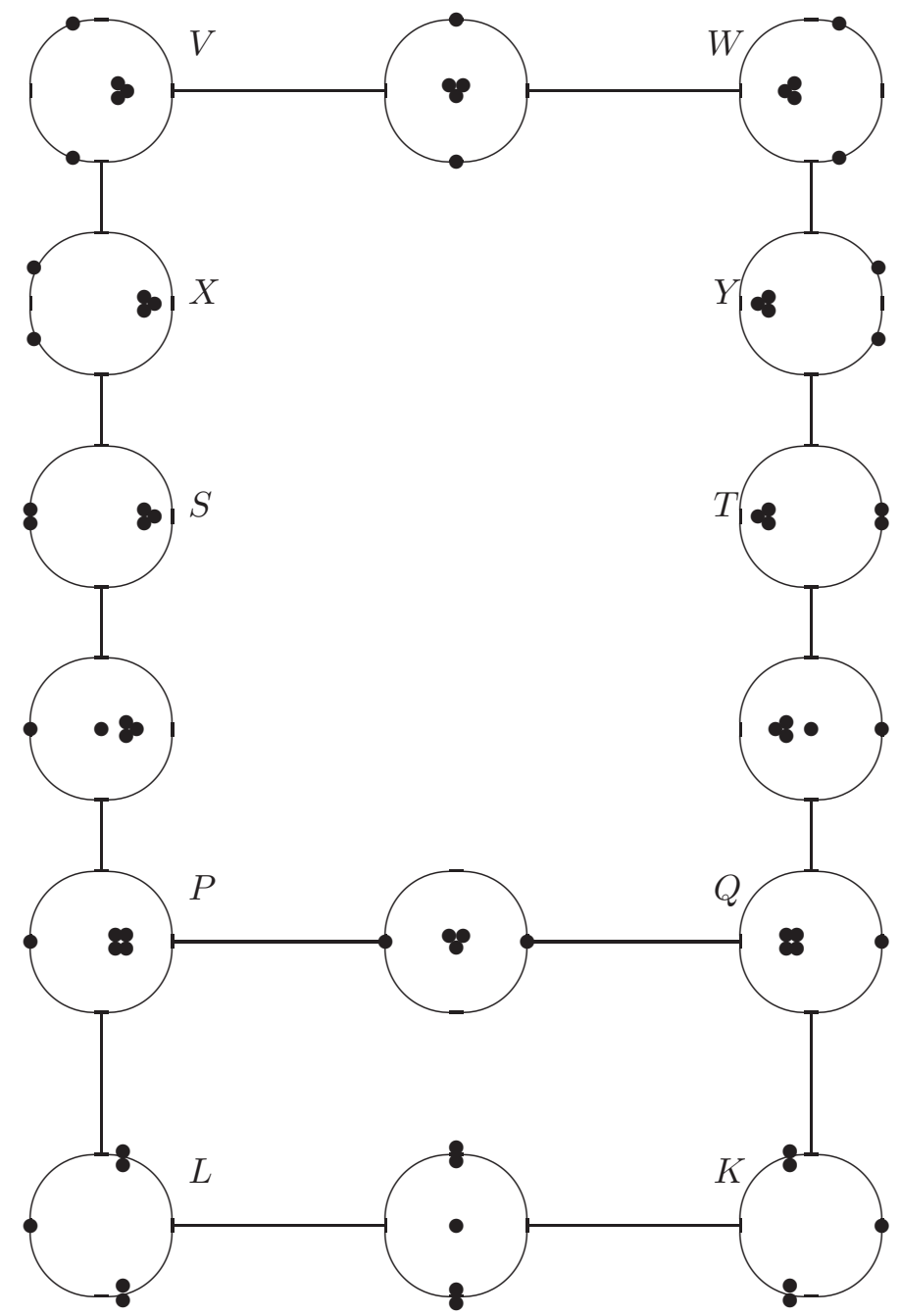

Рис. 2. Внутренняя топология множества существенного ветвления

не содержит оси $D$ пространства $\mathbb{R}^{4}$. Выбросим из нашего факторпространства $S^{3}$ две точки, соответствующие половинам этой оси. Оставшееся многообразие является тривиальным расслоением над $S^{2}$ со слоем $\mathbb{R}^{1}$, где $S^{2}$ можно рассматривать как фактор пространства полиномов вида $x^{5}+A x^{3}+B x^{2}+C x$ по действию группы $\mathbb{R}_{+}^{1}$ растяжений $\lambda: f(x) \mapsto \lambda^{-5} f(\lambda x)$.

Найдем пары точек множества $\Upsilon$ (т. е. орбит действия (4)), имеющих одинаковые проекции в $S^{2}$, т. е. отличающихся только свободными членами соответствующих полиномов.

3.3. Самопересечение проекции множества \& полиномов с трехкратными вещественными корнями. Найдем пары полиномов вида (2), 


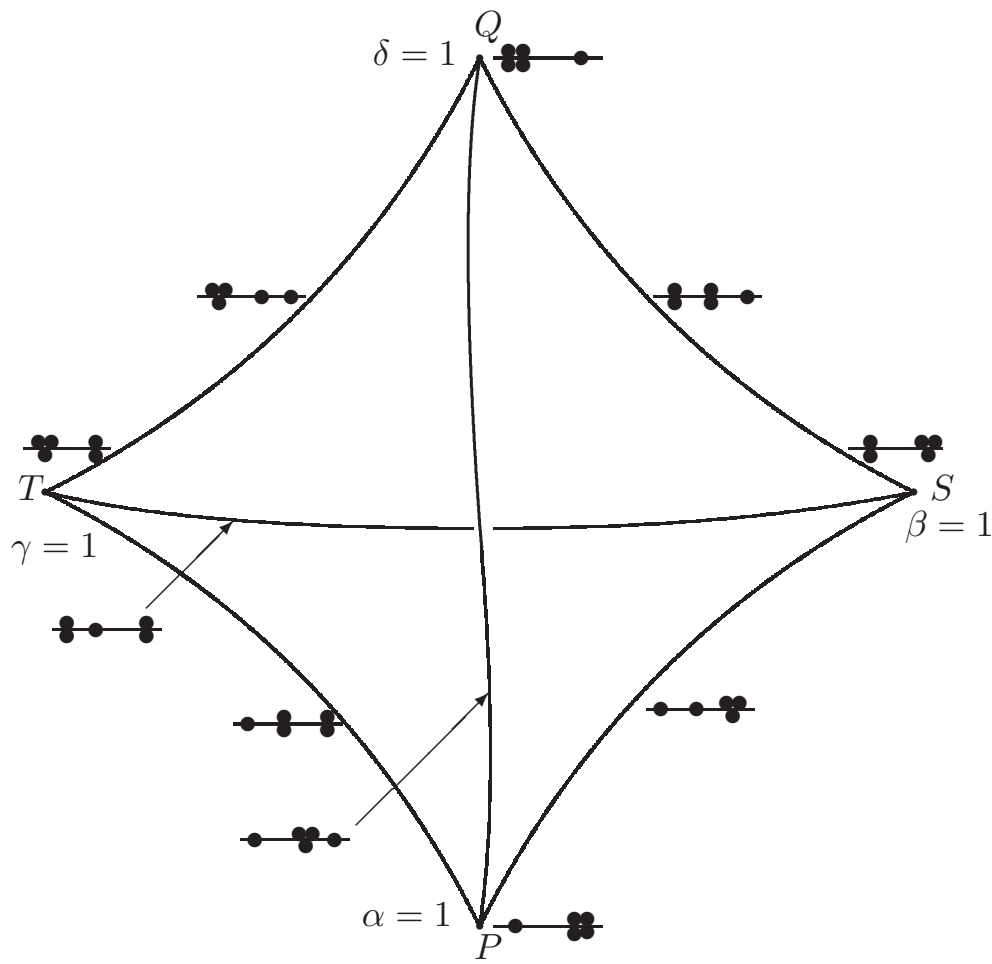

Рис. 3. Многочлены пятой степени с пятью вещественными корнями

имеющих трехкратные вещественные корни и отличающихся только свободными членами. Общая производная таких полиномов должна иметь два двукратных корня, т. е. равняться

$$
\rho(x-a)^{2}(x-\tilde{a})^{2}
$$

для некоторых $a, \tilde{a}, \rho$. Коэффициенты при мономах степени 5 и 4 искомого полинома (т. е. интеграла этой функции) вида (2) равны соответственно 1 и 0 , откуда $\rho=5, a+\tilde{a}=0$. Тогда искомые функции с точностью до выбора свободного члена равны

$$
x^{5}-\frac{10}{3} a^{2} x^{3}+5 a^{4} x .
$$

Чтобы найти, при каких значениях свободного члена они имеют трехкратный корень, приравняем их выражению

$$
(x-b)^{3}\left(x^{2}+3 b+c\right)=x^{5}+\left(c-6 b^{2}\right) x^{3}+\left(8 b^{3}-3 b c\right)+\left(3 b^{2} c-3 b^{4}\right)-b^{3} c .
$$

Равенство коэффициентов полиномов (5), (6) при мономах $x^{3}, x^{2}$ и $x$ дает условия $a^{2}=b^{2}, 3 c=8 b^{2}$, т. е. наши полиномы имеют вид $(x-b)^{3}\left(x^{2}+3 b x+\frac{8}{3} b^{2}\right)$, $b= \pm a$. Положительные и отрицательные значения $b$ дают две разные орбиты действия (4), т. е. две точки сферы $S^{3}$. Обозначим через $X$ (соответственно $Y$ ) ту точку, которая соответствует положительному (соответственно отрицательному) значению $b$. Дискриминант полинома $x^{2}+3 b x+\frac{8}{3} b^{2}$ отрицателен, значит, обе эти точки находятся в интервале $(S, T)$ множества $\Upsilon$, изображенном в верхней части рис. 2. При этом $X$ лежит в левой половине этого рисунка 
(где трехкратный корень положителен), а $Y$ - в правой. У полиномов, составляющих $X$, свободный член отрицателен, а у составляющих $Y$ положителен, следовательно, на диаграмме узла $\Upsilon$ в $S^{2}$ точка $Y$ лежит выше, чем $X$.

3.4. Фантомная кривая $\ddagger$ проектируется взаимно однозначно. Докажем, что подмножество в $\Upsilon$, состоящее из полиномов с парой мнимых сопряженных двукратных корней, проектируется в $S^{2}$ взаимно однозначно. Полиномы вида (2), имеющие такие корни, должны равняться

$$
\left(x^{2}+a x+b\right)^{2}(x-2 a) \equiv x^{5}+x^{3}\left(2 b-3 a^{2}\right)+x^{2}\left(-2 a b-2 a^{3}\right)+x\left(b^{2}-4 a^{2} b\right)-2 a b^{2}
$$

для некоторых $a, b$, таких, что

$$
a^{2}<4 b
$$

Допустим, что найдется другой полином такого же вида, т. е. равный

$$
\left(x^{2}+c x+d\right)^{2}(x-2 c) \equiv x^{5}+x^{3}\left(2 d-3 c^{2}\right)+x^{2}\left(-2 c d-2 c^{3}\right)+x\left(d^{2}-4 c^{2} d\right)-2 c d^{2},
$$

совпадающий с этим с точностью до свободного члена. Условие их совпадения дает систему уравнений

$$
2 b-3 a^{2}=2 d-3 c^{2}, \quad a b+a^{3}=c d+c^{3}, \quad b^{2}-4 a^{2} b=d^{2}-4 c^{2} d .
$$

Исключая неизвестные $b$ и $d$ и отбрасывая решения, приводящие к полному совпадению этих полиномов, получаем соотношение

$$
13 a^{2}+10 a c+13 c^{2}=0
$$

неразрешимое в вещественной области. Следовательно, эта часть множества действительно проектируется в $S^{2}$ без самопересечений.

3.5. Пересечение проекций кривых $\ddagger$ и $\$$. Теперь перечислим точки в $S^{2}$, в которые проектируются как «фантомные» точки ветвления (т. е. полиномы вида (7)), так и полиномы с трехкратными вещественными корнями. Полиномы последнего типа должны иметь вид

$$
(x-c)^{3}\left(x^{2}+3 c x+d\right) \equiv x^{5}+x^{3}\left(d-6 c^{2}\right)+x^{2}\left(8 c^{3}-3 c d\right)+x\left(3 c^{2} d-3 c^{4}\right)-c^{3} d .
$$

Приравнивая коэффициенты при $x^{3}, x^{2}, x$ у полиномов (11) и (7), получаем систему уравнений

$$
2 b-3 a^{2}=d-6 c^{2}, \quad-2\left(a b+a^{3}\right)=8 c^{3}-3 c d, \quad b^{2}-4 a^{2} b=3 c^{2} d-3 c^{4} .
$$

Исключая $b$ и $d$, приходим к соотношению на $a$ и $c$ :

$$
20 a^{6}+60 a^{5} c-75 a^{4} c^{2}-300 a^{3} c^{3}+240 a c^{5}-80 c^{6}=0 .
$$

Полагая $t=a / c$, получаем

$$
4 t^{6}+12 t^{5}-15 t^{4}-60 t^{3}+48 t-16 \equiv(t+2)^{3}(t-2)(2 t-1)^{2}=0 .
$$

Решение $t=-2$ дает

$$
a=-2 c, \quad b=c^{2}, \quad d=-4 c^{2} .
$$

Тогда полиномы (7) и (11) совпадают и равны

$$
x^{5}-10 c^{2} x^{3}+20 c^{3} x^{2}-15 c^{4} x+4 c^{5} \equiv(x-c)^{4}(x+4 c),
$$

т. е. это попросту общие концы $P, Q$ рассматриваемых интервалов множества $\Upsilon$.

Решение $t=1 / 2$ дает

$$
c=2 a, \quad b=-6 a^{2}, \quad d=9 a^{2} .
$$


Тогда полиномы (7) и (11) совпадают и равны

$$
x^{5}-15 a^{2} x^{3}+10 a^{3} x^{2}+60 a^{4} x-72 a^{5} \equiv(x-2 a)^{3}(x+3 a)^{2},
$$

т. е. это вершины $S$ и $T$ симплекса с рис. 3. Очевидно, что для этих точек не выполнено дискриминантное условие (8).

Решение $t=2$ дает

$$
a=2 c, \quad b=21 c^{2}, \quad d=36 c^{2} .
$$

Тогда полином (7) принимает вид

$$
x^{5}+30 c^{2} x^{3}-100 c^{3} x^{2}+105 c^{4} x-1764 c^{5},
$$

а полином (11) почти такой же, но со свободным членом $-36 c^{5}$.

Семейства таких полиномов, соответствующие положительным и отрицательным значениям $c$, определяют разные пары точек в $S^{3}$, проектирующиеся в разные точки в $S^{2}$. Обозначим через $K$ и $L$ фантомные (т. е. принадлежащие множеству $\ddagger)$, а через $V$ и $W$ - принадлежащие множеству \& полиномы из этих пар, отвечающие соответственно положительным и отрицательным значениям $c$. Тогда точка $V$ на диаграмме узла лежит выше чем $K$, а точка $L$ выше чем $W$.

Итак, мы имеем четыре интересные точки на интервале $(S, T)$, изображенном в верхней части рис. $2: Y$ - верхний и $X$ - нижний прообразы точки самопересечения проекции этого интервала в $S^{2}, V$ - прообраз точки пересечения проекций множеств $\ddagger$ и $\mathbf{s}$, описанной в предыдущем абзаце и соответствующей положительным значениям $c$, и $W$ - прообраз аналогичной точки, соответствующей отрицательным значениям $c$. При этом точки $X$ и $V$ лежат в левой части рис. 2, а точки $Y$ и $W$ - в правой.

Предложение 5. Эти точки расположены на отрезке $[S, T]$ в верхней части рис. 2 в следующем порядке: $S, X, V, W, Y, T$.

Доказательство. На этом отрезке аргумент того из мнимых корней полинома, который находится в верхней полуплоскости, монотонно убывает от $\pi$ к 0 . Легко проверяется, что у полиномов, реализующих наши точки, эти мнимые части упорядочены именно таким образом.

Согласно предыдущим вычислениям, полиномы $X$ и $Y$ (соответственно $K$ и $V$, соответственно $W$ и $L)$ совпадают с точностью до свободного члена, который в каждой из этих пар больше у второго ее элемента. Это завершает описание диаграммы проекции множества существенного ветвления $\Upsilon \cap S^{3}$ в $S^{2}$ с точностью до изотопии в $S^{2}$, в частности завершает доказательство теоремы 1.

\section{§4. Гомологии дополнения до множества существенного ветвления}

Теорема 2. Для любого $d$ и любой группљ $G=\mathbb{Z}$ или $\mathbb{Z}_{q}$

$$
H_{i}\left(\mathbb{R}^{d} \backslash \Upsilon, G\right) \simeq \bigoplus_{\substack{k \geqslant 0 \leqslant m, 3 k+4 m \leqslant d}} H_{i-k-m}\left(B\left(\mathbb{C}_{+}^{1}, m\right), \pm G\right),
$$

где $B\left(\mathbb{C}_{+}^{1}, m\right)$ - пространство всевозможсных подмножеств мощности $m$ в верхней полуплоскости $\mathbb{C}_{+}^{1} \equiv\{z: \operatorname{Im} z>0\}, \pm G-$ локальная система групп на этом пространстве, послойно изоморфная $G$, но меняющая ориентацию при переносе над путями, задающими нечетную перестановку $m$ точек. 
Участвующие в этой формуле пространства $B\left(\mathbb{C}_{+}^{1}, m\right)$ - классифицирующие пространства групп кос; их гомологии хорошо известны, см., например, [10].

Пример 1. При $d=5,6,7,8,9 u G \neq \mathbb{Z}_{2}$ полиномы Пуанкаре $G$-свободных частей групп $H_{*}\left(\mathbb{R}^{d} \backslash \Upsilon, G\right)$ равны соответственно $1+2 t, 1+2 t+t^{2}, 1+2 t+2 t^{2}$, $1+2 t+2 t^{2}, 1+2 t+2 t^{2}+t^{3}$. Для $d<8$ ( а если $G=\mathbb{Z}_{q}, q$ нечетно, то и для $d=8$ и 9) эти группы являются свободными $G$-модулями. При $d=8$ или 9 и $G=\mathbb{Z}$ (соответственно $\mathbb{Z}_{q}$ с четным $q \neq 2$ ) эта группа содержит дополнительно слагаемое $\mathbb{Z}_{2}$ в размерности 2 (соответственно в размерностях 2 и 3 ). Если $G=\mathbb{Z}_{2}$, то при $d=5,6,7$ полиномы Пуанкаре этих групп такие же, как выше; при $d=8$ и 9 эти полиномы равны соответственно $1+2 t+3 t^{2}+t^{3} u$ $1+2 t^{2}+3 t^{2}+2 t^{3}$.

Доказательство теоремы 2 следует стандартной схеме из [10], основанной на симплициальном разрешении множества $\Upsilon$. В частности, формула (13) peализуется следующим образом (ср. [10, §III.6]). Пусть $\gamma$ - произвольный цикл, представляющий некоторый элемент группы $H_{i-k-m}\left(B\left(\mathbb{C}_{+}^{1}, m\right), \pm G\right)$. Его носитель компактен, поэтому найдется такое $\varepsilon>0$, что для каждой точки этого цикла, т. е. набора из $m$ точек в $\mathbb{C}_{+}^{1}$, все эти $m$ точек находятся на расстояниях $\geqslant 5 \varepsilon$ друг от друга и от вещественной оси. Для каждой такой точки $\left(z_{1}, \ldots, z_{m}\right)$ рассмотрим в $B\left(\mathbb{C}_{+}^{1}, 2 m\right)$ вложенный $m$-мерный тор, состоящий из всевозможных $2 m$-конфигураций вида $\left(z_{1}+\varepsilon e^{i \alpha_{1}}, z_{1}-\varepsilon e^{i \alpha_{1}}, \ldots, z_{m}+\varepsilon e^{i \alpha_{m}}, z_{m}-\varepsilon e^{i \alpha_{m}}\right)$ со всевозможными $\alpha_{1}, \ldots, \alpha_{m} \in[0, \pi)$. Каждая точка такого тора определяет точку пространства $\mathbb{R}^{4 m} \backslash \Upsilon_{4 m}$, а именно вещественный полином степени $4 m$ с коэффициентом 1 при $x^{4 m}$, корни которого пробегают соответствующую $2 m$-конфигурацию и комплексно-сопряженную к ней; таким образом, наш тор оказывается вложен в $\mathbb{R}^{4 m} \backslash \Upsilon_{4 m}$. Объединение таких торов по всем $\left(z_{1}, \ldots, z_{m}\right) \in \gamma$ заметает некоторый $(i-k)$-мерный $G$-цикл $\Gamma$ в этом пространстве. Кроме этого рассмотрим стандартный $k$-мерный тор $T^{k} \subset \mathbb{R}^{3 k} \backslash \Upsilon_{3 k}$, состоящий из всевозможных полиномов вида

$\left((x-1)^{3}+\cos \left(\beta_{1}\right) \varepsilon^{2}(x-1)+\sin \left(\beta_{1}\right) \varepsilon^{3}\right) \cdots\left((x-k)^{3}+\cos \left(\beta_{k}\right) \varepsilon^{2}(x-k)+\sin \left(\beta_{k}\right) \varepsilon\right)$,

где $\varepsilon$ мало, а $\beta_{1}, \ldots, \beta_{k}$ независимо пробегают отрезок $[0,2 \pi]$. Прямое произведение $\Gamma \times T^{k}$ вложено в $\mathbb{R}^{d} \backslash \Upsilon$ : каждой паре точек $\phi \in \Gamma, \psi \in T^{k}$ соответствует полином

$$
\phi \times \psi \times(x-N)(x-2 N) \cdots(x-(d-3 k-4 m) N),
$$

где $N$ - достаточно большое число, превосходящее и $k+1$, и максимальное значение абсолютных величин всех точек в $\mathbb{C}_{+}^{1}$, участвующих в конфигурациях, составляющих цикл $\gamma$. Образ этого вложения определяет $i$-мерный цикл, гомологический класс которого - это и есть искомый класс в $H_{i}\left(\mathbb{R}^{d} \backslash \Upsilon, G\right)$, соответствующий $\gamma$ в силу равенства (13).

Симплициальное разрешение для $\Upsilon$, строящееся по схеме из [10], дает спектральную последовательность для вычисления групп $H_{i}\left(\mathbb{R}^{d} \backslash \Upsilon, G\right)$, сумма членов $E_{p, q}^{1}$ которой равна правой части в $(13)$, а только что описанная реализация этих слагаемых циклами доказывает стабилизацию в этом члене.

Замечание 3. В силу теоремы двойственности Александера гомологии пространства $\mathbb{R}^{d} \backslash \Upsilon$ с постоянными коэффициентами сводятся к внутренней топологии множества $\Upsilon$ и ничего не говорят о характере его заузленности в $\mathbb{R}^{d}$. 
Для оценки рода Шварца наиболее интересны не целочисленные гомологии пространств $\mathbb{R}^{d} \backslash \Upsilon$, а гомологии с коэффициентами в подходящих локальных системах, ассоциированных с накрытием $\Theta_{d}$, см. [7], [9]. В частности, интересен гомотопический тип этих пространств.

Также, аналогично [1], топологические инварианты множества существенного ветвления (и самого этого ветвления) могут использоваться как препятствия к индуцированию вещественных алгебраических функций друг из друга.

Наконец, упомяну работы [4], [6], примыкающие к данной проблематике.

\section{ЛитеРАТУРА}

[1] В. И. Арнольд, Топологические инварианты алгебраических функиий. II, Функц. анализ и его прил., 4:2 (1970), 1-9.

[2] В. И. Арнольд, В. А. Васильев, В. В. Горюнов, О. В. Ляшко, Особенности. І. Локальная и глобальная теория, Итоги науки и техники. Современные проблемы математики. Фундаментальные направления, т. 6, ВИНИТИ, М., 1988.

[3] В. И. Арнольд, Особенности каустик и волновых фронтов, Фазис, М., 1996.

[4] M. G. Khovanov, Real $K(\pi, 1)$-arrangements from finite root systems, Math. Res. Letters, 3:2 (1996), 261-274.

[5] Ф. Клейн, Лекиии об икосаэдре и решении уравнений пятой степени, Наука, М., 1989.

[6] E. J. N. Looijenga, The discriminant of a real simple singularity,, Compositio Math., 37:1 (1978), 51-62.

[7] А. С. Шварц, Род расслоенного пространства, Труды ММО, 10 (1961), 217-272.

[8] S. Smale, On the topology of algorithms, J. Complexity, 3:2 (1987), 81-89.

[9] В. А. Васильев, Когомологии групп кос и сложность алгоритмов, Функц. анализ и его прил., 22:3 (1988), 15-24.

[10] В. А. Васильев, Топология дополнений к дискриминантам, Фазис, М., 1997.

[11] В. А. Васильев, Топологическая сложность и вещественность, Матем. заметки, 60:5 (1996), 670-680.

[12] V. A. Vassiliev, Topological complexity and Schwarz genus of general real polynomial equation, Moscow Math. J., 11 (2011).

Математический институт им. В. А. Стеклова РАН Национальный исследовательский университет Поступило в редакцию «Высшая школа экономики», факультет математики e-mail:vva@mi.ras.ru 